\title{
Which is the best route of administration for cell therapy in experimental model of small-for size syndrome in rats?
}

\author{
Lucas Souto Nacif ${ }^{\text {I }}$ Amanda Olivotti Ferreira ${ }^{\mathrm{II}}$, Durvanei Augusto Maria ${ }^{\mathrm{III}}$, Márcia Saldanha Kubrusly ${ }^{\mathrm{IV}}$, Nilza Molan ${ }^{\mathrm{V}}$, Eleazar \\ Chaib $^{\text {VI }}$ Luiz Carneiro D'Albuquerque ${ }^{\mathrm{VII}}$, Wellington Andraus ${ }^{\mathrm{VIII}}$
}

\author{
DOI: http://dx.doi.org/10.1590/S0102-86502015002000003
}

${ }^{\mathrm{I}}$ Fellow PhD degree and MSc degree, Postgraduate Program in Medicine (Science in Gastroenterology), Department of Gastroenterology, School of Medicine, Medical Investigation Laboratory (LIM 37), University of Sao Paulo (USP), Brazil. Carried out all experimental and surgical procedures, acquisition of data, statistical analysis, drafted the manuscript.

IIMSc degree, Biochemistry, Laboratory of Biochemistry and Biophysics, Biochemistry in Biotechnology Center, Butantan Institute, Sao Paulo-SP, Brazil. Acquisition of data, designed the preparation and characterization of stem cells of the liver.

IIIAssociate Professor, Laboratory of Biochemistry and Biophysics, Biochemistry in Biotechnology Center, Butantan Institute, Sao Paulo-SP, Brazil. Designed the preparation and characterization of stem cells of the liver, critical revision.

${ }^{\mathrm{IV}}$ Fellow PhD degree, Postgraduate Program in Medicine (Surgical Gastroenterology), Biochemistry in Liver and Gastrointestinal Transplant Division, Department of Gastroenterology, School of Medicine, LIM 37, USP, Sao Paulo-SP, Brazil. Statistical analysis, drafted the manuscript.

vBiochemistry, Liver and Gastrointestinal Transplant Division, Department of Gastroenterology, School of Medicine, LIM 37, USP, Sao Paulo-SP, Brazil. Laboratory analysis, technical procedures.

${ }^{\mathrm{VI}}$ Associate Professor, Liver and Gastrointestinal Transplant Division, Department of Gastroenterology, School of Medicine, LIM 37, USP, Sao PauloSP, Brazil. Design of the study, manuscript writing, critical revision.

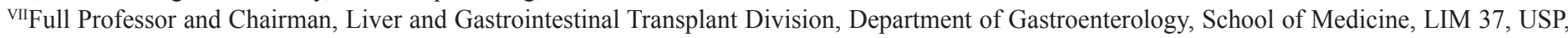
Sao Paulo-SP, Brazil. Conception and design of the study, manuscript writing, critical revision.

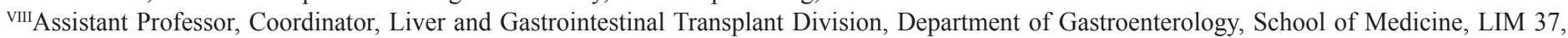
USP, Sao Paulo-SP, Brazil. Technical procedures, manuscript writing, critical revision.

\begin{abstract}
PURPOSE: To evaluate which is the best route of administration for cell therapy in experimental rat model of small-for size syndrome. METHODS: A total of 40 rats underwent partial hepatectomy (70\%) that induces the small-for-size syndrome and were divided into four groups of route administration: intravenous, intraperitoneal, enteral and tracheal. The small-for-size syndrome model was designed with extended partial hepatectomy $(70 \%)$. The animals were divided into four groups of routes administration: intravenous $(\mathrm{n}=10)$ intravenously through the dorsal vein of the penis; intraperitoneal $(n=10)$ - intraperitoneally in the abdominal cavity; enteral $(n=10)$ - oroenteral with the placement of a number 4 urethral probe and maintained at third duodenal portion; tracheal ( $\mathrm{n}=10)$ - after tracheal intubation. We track the animals and monitor them for 21 days; during this follow-up we evaluated the result of cell therapy application tracking animals using ultrasound, radiography and PET-scan. Statistical analysis was performed using GraphPad Prism Software ${ }^{\circledR}$. Differences were considered significant with the $\mathrm{p}<0.05$. Data are presented as the median and variation for continuous variables. Comparisons between groups were made using analysis of the imaging test by the researchers.

RESULTS: All four groups underwent partial hepatectomy of $70 \%$ liver tissue targeting the same weight of resected liver. Initially the PET-scan tests showed similarity in administered cells by different routes. However, in few days the route of intravenous administration showed to be the most appropriated to lead cells to the liver followed by enteral. The tracheal and peritoneal routes were not as much successful for this goal.
\end{abstract}

CONCLUSION: The intravenous route is the best one to cell therapy in experimental rat model of small-for size-syndrome.

Key words: Liver Regeneration. Disease Models, Animal. Stem Cells. Rats. 


\section{Introduction}

The Small-for-Size Syndrome (SSS) is seen more often if the transplanted segment of the donor is $<30 \%$ liver or $<0.8 \%$ of body weight of the recipient ${ }^{1}$. The SSS is a complex process resulting primarily from ischemia-reperfusion injury (IRI) and portal hypertension associated with mismatch between the graft and recipient size. In the initial period, the molecular events associated with subsequent apoptosis, necrosis, proliferation and regeneration appeared in specific patterns of protein expression ${ }^{2-4}$.

The diagnosis of SSS graft is generally based on persistent hyperbilirubinemia, coagulopathy, massive ascites during subacute phase post-transplant or extensive hepatic resection ${ }^{2,5}$. Living donor liver transplantation can reduce the discrepancy between the number of patients on the waiting list for transplantation and the number of donor organs available.

Stem cells have three properties: self-renewal, proliferation and differentiation. They have the potential to generate large numbers of mature cells continuously throughout life $\mathrm{e}^{6,7}$. The term refers to cells having ability to generate daughter cells with characteristics similar to the mother cell, as well as differentiate into specialized cells ${ }^{6,7}$. Stem cells are able to differentiate into all cell types and can be used in replacement therapies for various diseases. Embryonic and fetal liver cell lines can be an important tool for cell therapy in patients with liver disease source, as they have a high rate of differentiation into hepatocytes and bile duct cells. Although there are numerous published papers on the administration of stem cells, none of them evaluate the best route of administration. The aim of this study was to evaluate which is the best route of administration for cell therapy in experimental rat model of liver regeneration on small-for size-syndrome.

\section{Methods}

The study was designed in accordance with the Guide for the Care and Use of Laboratory Animals of University of São Paulo. This project was approved by the Ethics Committee, for the the Guidelines of Animal Experimentation of the University of São Paulo School of Medicine, São Paulo, SP, Brazil.

A total of 40 rats male Sprague-Dawley (SD) rats (200 $250 \mathrm{~g}$, Biotechnology Center, Butantan Institute) were housed at $19^{\circ} \mathrm{C} \pm 3^{\circ} \mathrm{C}$ with a $12-\mathrm{h}(06: 00-18: 00$ hours $)$ artificial light cycle. Two or three animals from the same treatment group were housed per cage. The animals had free access to tap water and standard food during the entire experiment. Food intake was not measured.

\section{Study design}

The animals underwent an extended partial hepatectomy $(70 \%)$ that induce the small-for-size syndrome and were divided into four groups of routes administration (Figure 1): intravenous $(n=10)$ - intravenously through the dorsal vein of the penis; intraperitoneal $(n=10)$ - intraperitoneally in the abdominal cavity; enteral $(n=10)$ - oroenteral with the placement of a number 4 urethral probe and maintained at third duodenal portion; tracheal $(\mathrm{n}=10)$ - after tracheal intubation (Figure 2). We track the animals and monitor them for 21 days; during this follow-up we evaluated the result of cell therapy application tracking animals using ultrasound, radiography and PET-scan. All procedures began with the intraperitoneal anesthesia using 5\% ketamine hydrochloride 30 $\mathrm{mg} / \mathrm{kg}\left(\operatorname{Ketalar}^{\circledR}\right.$, Cristália). Animals were kept warm with a $45 \mathrm{~W}$, $127 \mathrm{~V}$ halogen bulb and body temperature was monitored using a rectal digital thermometer (YSI 4000A Precision Thermometer, USA) and maintained between $35^{\circ} \mathrm{C}$ and $37^{\circ} \mathrm{C}$.

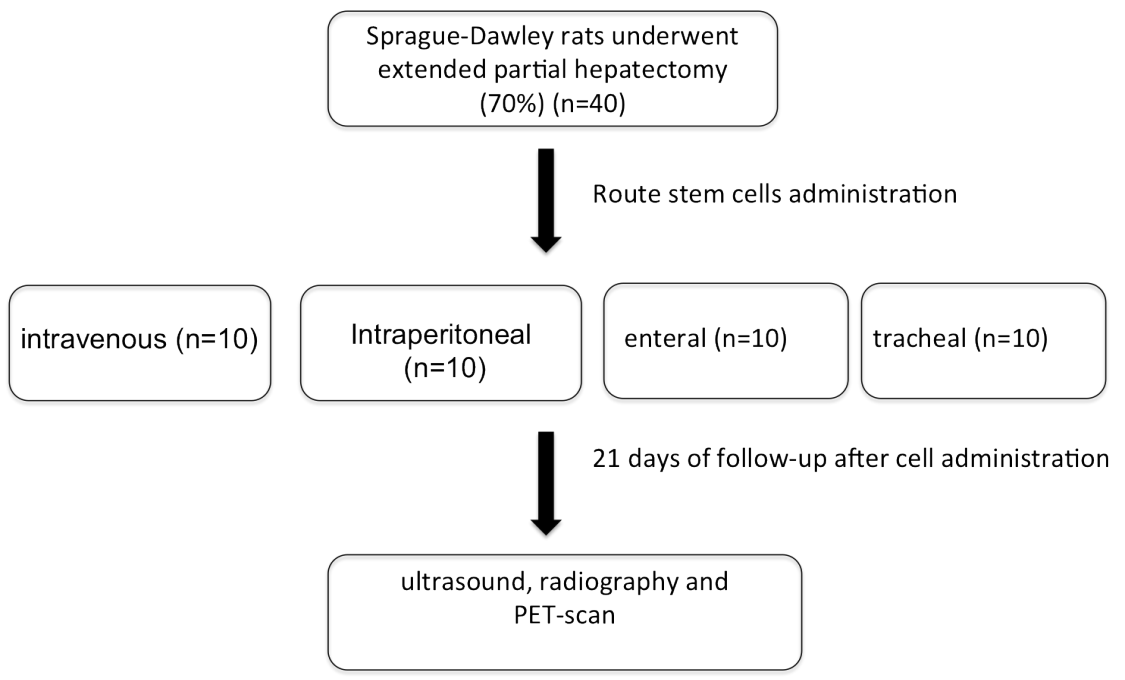

FIGURE 1 - The study design for four groups of different routes administration in experimental small-for-size syndrome. 


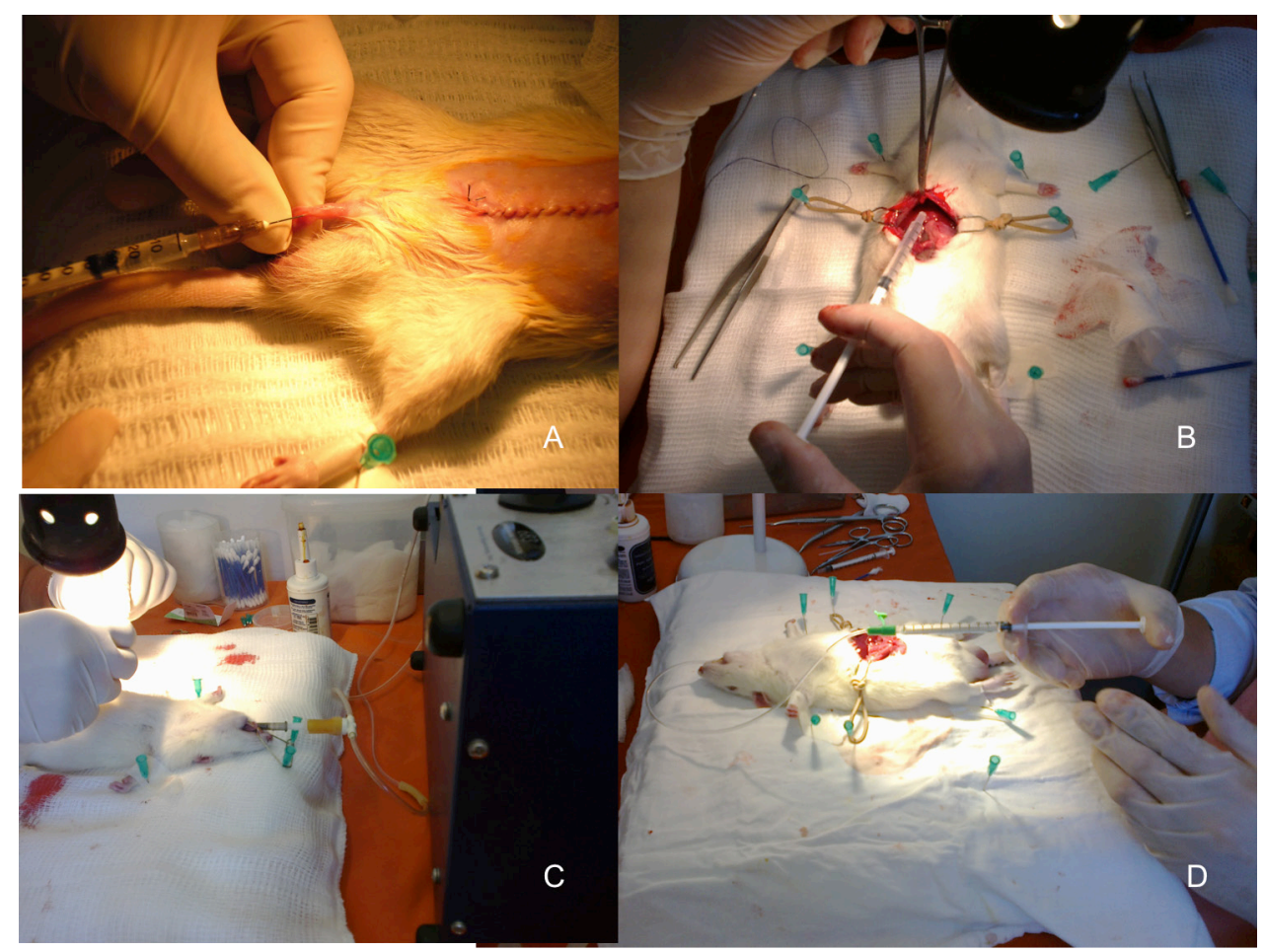

FIGURE 2 - The four groups of different routes of administration in experimental small-for-size syndrome. A. Intravenous; B. Intraperitoneal; C. Enteral; D. Tracheal.

All animals were anesthetized and underwent a surgical intervention with median laparotomy extending for approximately $4 \mathrm{~cm}$ from the xiphoid process; the left and median liver lobes were removed after the central ligature with 4-0 cotton. The right upper, middle and lower lobes were ligated in the common right lobe pedicle of the liver using cotton yarn performing ischemia and subsequent necrosis. Both lobes of the liver and the liver tissue pieces around the intrahepatic portion of the inferior vena cava remained together. These two hepatic lobes represent about 30\% of the total liver mass (Figure 3). The remnant liver was confirmed with weight measurements. The abdominal incision was sutured with Mononylon ${ }^{\circledR} 4.0$.

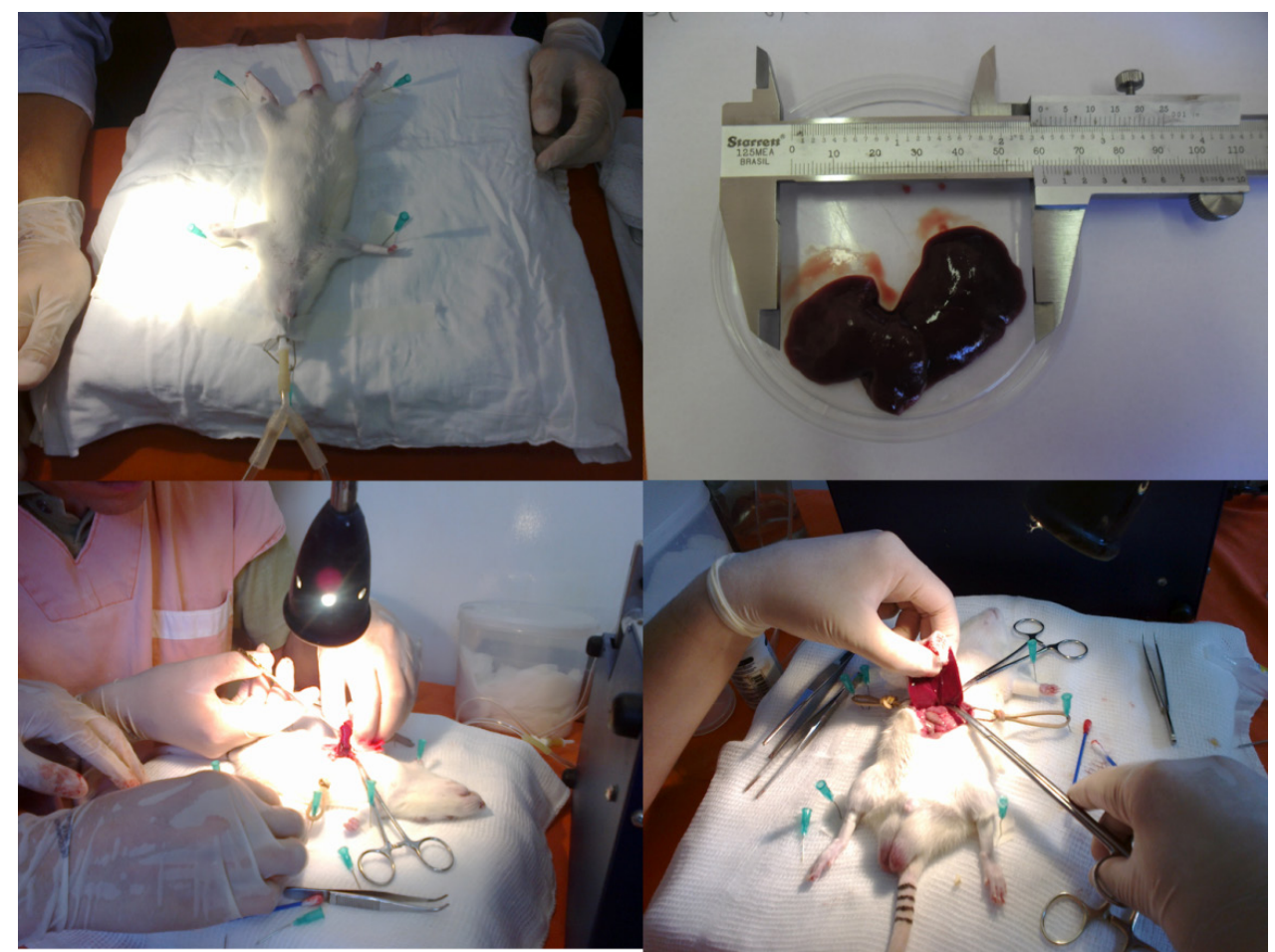

FIGURE 3 - Surgical technique of experimental small-for-size syndrome in rats. 


\section{Incorporation of Propidium Iodide (PI) and reading sample}

The fixed and previously stored samples were centrifuged at $2000 \mathrm{rpm}$ for 5 minutes. The supernatant was discarded, the cells resuspended in $1 \mathrm{ml}$ flow cytometry buffer and centrifuged again. After centrifugation, the supernatant was discarded and the cells resuspended in PI solution prepared from $5 \mathrm{ml} \mathrm{PBS} \mathrm{(sodium} \mathrm{phosphate}$ buffer $\mathrm{pH} 7.4)$ to which were added $1005 \mu \mathrm{L}$ Triton $(0.01 \% \mathrm{v} / \mathrm{v})$ and $50 \mu \mathrm{L}$ of RNAse $(2 \mathrm{mg} / \mathrm{mL})$ and $20 \mu \mathrm{L}$ of propidium iodide $(5 \mathrm{mg} / \mathrm{mL})$. Then, if the held data acquisition FACSCalibur flow cytometer on 10.000 events for analysis and Win Mdi 2.8 program was used.

\section{Evaluation of routs administration of stem cell in rats}

Initially, we performed the analysis in the seventh day after surgery; follow by second analysis in fifteenth day and subsequent twentieth day for establishing and detecting the stem cell by PET-scan, ultrasonography and radiography for each route administration group.

\section{Literature review}

A literature review was performed with the Mesh-terms "Liver regeneration"; "Disease models, Animal"; "Stem cells" and "Rats". The electronic search was held of the Medline-PubMed, in
English. The search was completed in September 2014.

\section{Statistics}

Statistical analysis was performed using GraphPad Prism Software ${ }^{\circledR}$. Differences were considered significant with the $p<0.05$. Data are presented as the median and variation for continuous variables. Comparisons between groups were made using analysis of the imaging test by the researchers.

\section{Results}

A total of 40 rats underwent partial hepatectomy that induces the small-for-size syndrome and the four groups of routes administration were evaluated in three moments (seventh, fifteenth and twenty-first day). They were carried out as follows: intravenous group $(n=10)$, intraperitoneal group $(n=10)$, enteral group $(\mathrm{n}=10)$ and tracheal group $(\mathrm{n}=10)$.

All four groups underwent partial hepatectomy of $70 \%$ liver tissue targeting the same weight of resected liver. The routes of administration for cell therapy in experimental rat model of small-for size syndrome for each group were evaluated using ultrasound, radiography and displayed below the PET-scan (Figure 4). During follow-up of twenty-one days, the cells remained alive after administration in all groups showed by PET-scan.

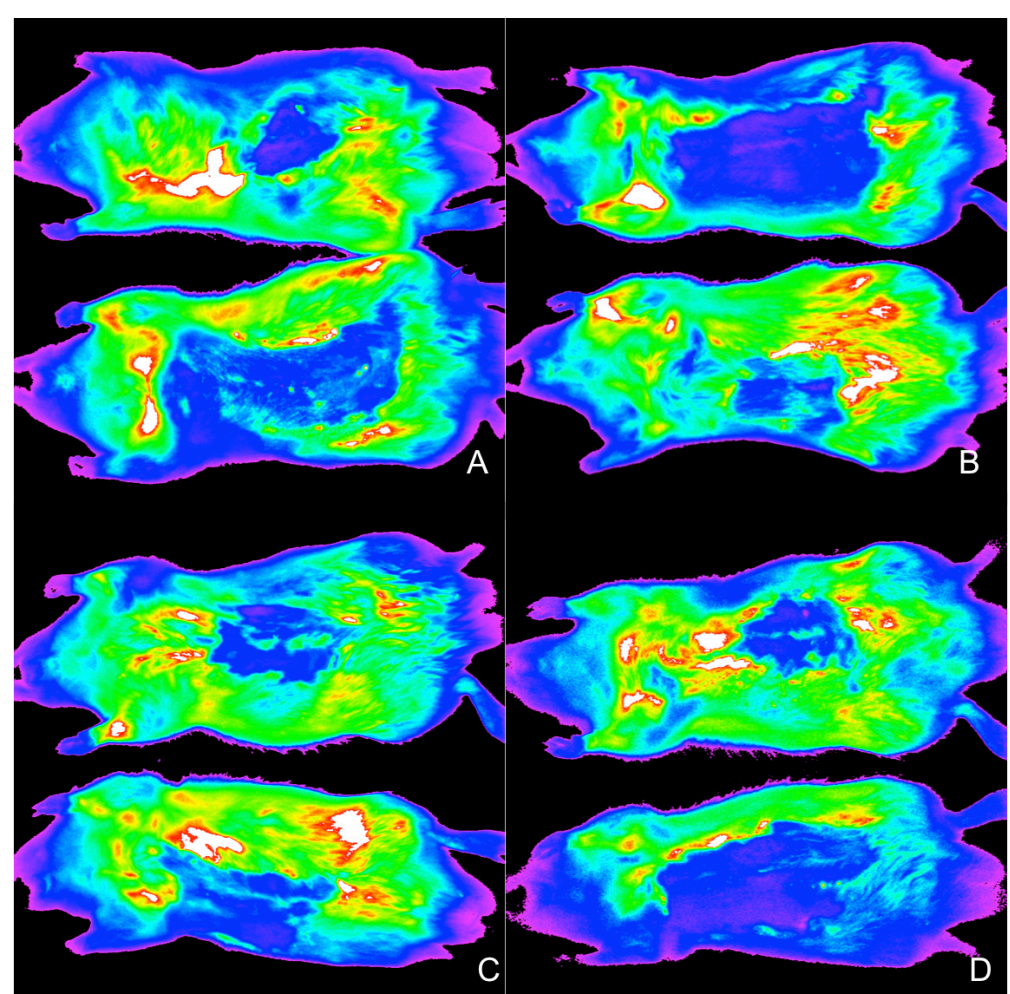

FIGURE 4 - PET-scan evaluation of the route of administration for cell therapy in experimental rat model of small-for size-syndrome. A. Intravenous; B. Peritoneal; C. Tracheal; D. Enteral.

Note: The animal above is the twenty-first day and down the fifteenth day post administration. 
Initially it was observed in PET-scan tests a similarity between administered cells by different routes. However, after few days the intravenous route showed to be more appropriated to lead cells to the liver, followed by enteral route. The tracheal and peritoneal routes were not as much efficient for this ultimate goal. The evaluation by ultrasound and radiography of the rats, in the different groups showed no differences to show the marked stem cell (Figure 5).
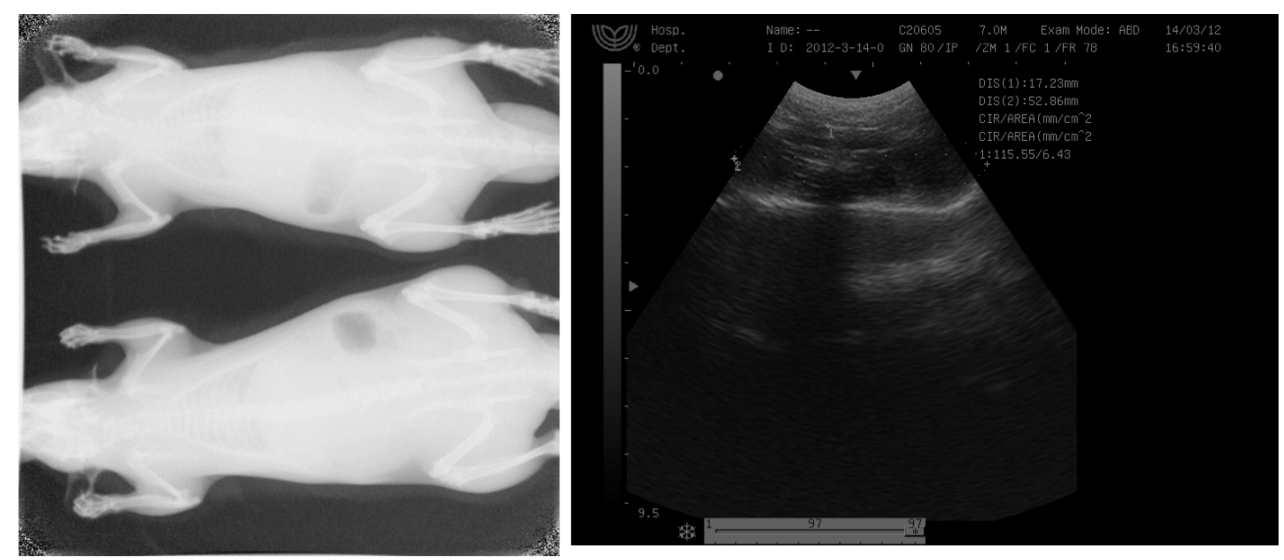

FIGURE 5 - The ultrasound and radiography evaluation the route of administration for cell therapy in experimental rat model of small-for size syndrome.

Note: The animal above is the twenty-first day post administration.

In the laboratory analysis, we observed an elevation of liver enzymes (AST and ALT), slight change of total and direct bilirubin, and extending the coagulation time. Forty percent of the cases presented incoagulable value in the sample (Table 1).

A literature review was performed with the Mesh-terms: "Liver regeneration"; "Disease models, Animal"; "Stem cells" and "Rats" in order to see the administration route used by the authors in this specific field. The initial review search showed 69 articles in Medline-PubMed with the specific Mesh-terms and we analyzed the methods used in each abstract as showed in Figure 6.

TABLE 1 - Findings of the laboratory biochemical.

\begin{tabular}{ccc}
\hline $\begin{array}{c}\text { Parameters } \\
\text { AST(U/L) }\end{array}$ & 150.44 & (range, 122-210) \\
ALT(U/L) & 45.44 & (range, 26-70) \\
TB (U/L) & 0.065 & (range, 0.01-0.1) \\
DB (U/L) & 0.021 & (range, $0.01-0.04)$ \\
AP (U/L) & 121.15 & (range, 28.4-255) \\
GGT (U/L) & 1.3 & (range, $0.8-2.1$ ) \\
PT (\%)* & 65.2 & (range, 27 - 81) \\
INR range* & 1.53 & (range, 1.14-2.86) \\
\hline Note: The median and variation. AST, aspartate transaminase; ALT, alanine trans- \\
aminase; TB, total bilirubin; BD, direct bilirubin; GGT, gamma glutamyl transfer- \\
ase; AP, alkaline phosphatase; PT, prothrombin time; INR range. *Forty percent of \\
the cases presented incoagulable value in the sample and INR>5.
\end{tabular}

Note: The median and variation. AST, aspartate transaminase; ALT, alanine transaminase; TB, total bilirubin; BD, direct bilirubin; GGT, gamma glutamyl transferase; AP, alkaline phosphatase; PT, prothrombin time; INR range. *Forty percent of the cases presented incoagulable value in the sample and INR $>5$.

iterature review search 69 articles

Route stem cells administration

\begin{tabular}{|c|c|c|c|c|c|c|c|}
\hline $\begin{array}{c}\text { intravenous } \\
(n=25)\end{array}$ & $\begin{array}{c}\text { Intraperitoneal } \\
(n=5)\end{array}$ & enteral $(n=2)$ \\
*gavage tube & tracheal $(n=0)$ & $\begin{array}{c}\text { Subcutaneous } \\
(n=1)\end{array}$ \\
\hline
\end{tabular}

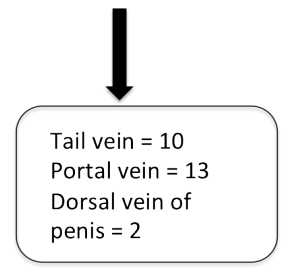

Excluded articles:

- Non English articles $=2$

- Without cell therapy $=12$

- Intraosseous $=2$

- Articles before $2000=10$

- Clinic review $=5$

FIGURE 6 - The literature review performed with the Mesh-terms: Liver regeneration; Disease models, Animal; Stem cells and Rats showed 69 articles in Medline-PubMed. 


\section{Discussion}

To our knowledge, this is the first study that evaluates the routs of stem cells administration in an experimental rat model. Although authors use published references to copy methods, it is indeed important to know which is the best route to cell therapy administration or transplantation in experimental models of liver regeneration. All four groups underwent partial hepatectomy of $70 \%$ targeting the same weight of resected liver. Initially, we observed in PET-scan tests a similarity in administered cells by different routes, but after 21 days the intravenous route administration showed to be the best one, with a bigger amount of cells in the liver site.

It is interesting to notice that the enteral route was better than peritoneal and tracheal routes, and it showed to be also a good route of administration. Other important point is that the enteral route of administration (gavage tube) was placed after the stomach, preventing the acid acting on the cells.

It is remarkable that the tracheal route group showed a representative amount of viable marked stem cells in the liver. Although we did not find any study using this way of administration in our review, this is also an option for specific studies, and it demonstrates the high absorption capacity of the animal in different sites when stem cells are administrated. By the literature review with 69 articles in Medline-PubMed, the most common route of infusion was intravenous in $62 \%$ of specific articles, followed by intraesplenic (12\%) and intraperitoneal (12\%).

Salomone et al. $^{6}$ showed the efficacy of stem cell transplantation in rats with acetaminophen liver injury on Sprague-Dawley rats that were administrated $300 \mathrm{mg} / \mathrm{kg}$ of acetaminophen intraperitoneally. The therapy was performed in six rats that received the infusion of stem cells via the caudal vein. They injected around 200.000 cells suspended in $1 \mathrm{~mL}$ of saline solution and six rats were administrated only saline. In this study, as an example, the authors used the intravenous administration.

The hepatocyte transplantation in animal models showed the intraportal route has been used in animal models of metabolic liver disorders. However, the peritoneal cavity and subcutaneous tissues have been investigated and these sites do not efficiently support cell viability for long periods because the transplanted cells have no direct and instant access to oxygen and nutrients ${ }^{8}$. Another study demonstrated the contribution of mature hepatocytes to small hepatocyte-like progenitor cells in retrorsine-exposed rats with chimeric livers that was administrated via intraportal ${ }^{7}$.
The spleen has proven to be also a good site for hepatocyte engraftment. The hepatocytes infused into the portal vein or infused into the spleen undergo blood flow-mediated translocation to the hepatic sinusoids. However, a large proportion remains in the spleen, which can serve as a site for long-term survival and function of engrafted hepatocytes. Intrasplenic hepatocyte transplantation has been performed in animal models of chronic liver failure ${ }^{8}$.

Ultimately, the intravenous route of administration was more appropriate to aim in the liver place, followed by enteral. All four routes tested showed to lead cells to the liver even the tracheal route. The intravenous route, as the most used, confirmed to be the best route for cells administration in rats models for liver regeneration.

\section{Conclusion}

The intravenous route is the best one to cell therapy in experimental rat model of small-for size-syndrome.

\section{References}

1. Nishizaki T, Ikegami T, Hiroshige S, Hashimoto K, Uchiyama H, Yoshizumi T, Kishikawa K, Shimada M, Sugimachi K.Small graft for living donor liver transplantation. Ann Surg. 2001;233:575-80. PMID: 11303141

2. Wu J, Tang Q, Shen J, Yao A, Wang F, Pu L, Yu Y, Li X, Li G, Zhang F, Sun B, Kong L, Li D, Zhang Y, Guo X, Wang X. Comparative proteome profile during the early period of small-for-size liver transplantation in rats revealed the protective role of Prdx5. J Hepatol. 2010 Jul;53(1):73-83. doi: 10.1016/j.jhep.2010.01.032.

3. Di Domenico S, Santori G, Traverso N, Balbis E, Furfaro A, Grillo F, Gentile R, Bocca B, Gelli M, Andorno E, Dahame A, Cottalasso D, Valente U. Early effects of portal flow modulation after extended liver resection in rat. Dig Liver Dis. 2011 Oct;43(10):814-22. doi: 10.1016/j.dld.2011.05.018.

4. Hessheimer AJ, Escobar B, Muñoz J, Flores E, Gracia-Sancho J, Taurá P, Fuster J, Rimola A, García-Valdecasas JC, Fondevila C. Somatostatin therapy protects porcine livers in small-for-size liver transplantation. Am J Transplant. 2014 Aug;14(8):1806-16. doi: 10.1111/ajt.12758.

5. Li J, Liang L, Ma T, Yu X, Chen W, Xu G, Liang T. Sinusoidal microcirculatory changes after small-for size liver transplantation in rats. Transplant Int. 2010 Sep;23(9):924-33. doi: 10.1111/j.14322277.2010.01058.x.

6. Salomone F, Barbagallo I, Puzzo L, Piazza C, Li Volti G. Efficacy of adipose tissue-mesenchymal stem cell transplantation in rats with acetaminophen liver injury. Stem Cell Res. 2013 Nov;11(3):103744. doi: 10.1016/j.scr.2013.07.003.

7. Chen $\mathrm{YH}$, Chang $\mathrm{MH}$, Chien $\mathrm{CS}, \mathrm{Wu} \mathrm{SH}, \mathrm{Yu} \mathrm{CH}$, Chen HL. Contribution of mature hepatocytes to small hepatocyte-like progenitor cells in retrorsine-exposed rats with chimeric livers. Hepatology. 2013 Mar;57(3):1215-24. doi: 10.1002/hep.26104.

8. Weber A, Groyer-Picard MT, Franco D, Dagher I. Hepatocyte transplantation in animal models. Liver Transplant. 2009 Jan;15(1):7-14. doi: 10.1002/lt.21670. 


\section{Correspondence:}

Wellington Andraus

Divisão de Transplantes de Fígado e Órgãos do Aparelho Digestivo

Departamento de Gastroenterologia-USP

Rua Dr. Enéas de Carvalho Aguiar, 255/9º andar, salas 9113/9114

05403-900 São Paulo - SP Brasil

Tel.: (55 11)2661-3323

Fax: (55 11)2661-9008

wellington@usp.br

Received: Oct 28, 2014

Review: Dec 26, 2014

Accepted: Jan 20, 2015

Conflict of interest: none

Financial source: none

${ }^{1}$ Research performed at Liver and Gastrointestinal Transplant Division, Department of Gastroenterology, School of Medicine, Medical Investigation Laboratory (LIM 37), University of Sao Paulo (USP) and Biotechnology Center, Butantan Institute, Sao Paulo-SP, Brazil. Part of PhD degree thesis, Postgraduate Program in Medicine (Science in Gastroenterology). Tutor: Wellington Andraus. 